\title{
Atherosclerotic Carotid Vulnerable Plaque and Subsequent Stroke: A High-Resolution MRI Study
}

\author{
Tianli Gao ${ }^{\mathrm{a}, \mathrm{b}}$ Zhuo Zhang ${ }^{\mathrm{b}}$ Wei Yu ${ }^{\mathrm{c}}$ Zhaoqi Zhang ${ }^{c}$ Yongjun Wang ${ }^{\mathrm{a}}$ \\ a Department of Neurology, Beijing Tiantan Hospital, and Departments of ${ }^{\mathrm{b}}$ Neurology and ${ }^{\mathrm{c}}$ Radiology, \\ Beijing Anzhen Hospital, Capital Medical University, Beijing, China
}

\section{Key Words}

Atherosclerosis $\cdot$ Carotid arteries $\cdot$ Stroke $\cdot$ Magnetic resonance imaging

\begin{abstract}
Background: High-resolution contrast-enhanced magnetic resonance imaging (CEMRI) has been proven to be an effective tool for the identification of carotid atherosclerotic vulnerable plaque, such as a large lipid core and thin fibrous cap. The aim of this study was to evaluate the relationship between carotid plaque characteristics and the types of stroke in patients who had carotid artery (CA) stenosis $\geq 50 \%$. Methods: 102 consecutive subjects (mean age $67.2 \pm 10.2$ years; 73 males) who initially had ischemic stroke or asymptomatic CA stenosis from 50 to $100 \%$ diagnosed by ultrasound were included in this study. Carotid CEMRI, brain MRI and magnetic resonance angiography were performed to understand the infarct patterns and to exclude intracranial artery stenosis. The modified American Heart Association (AHA) plaque classification was used in our study. Results: Our study demonstrated that 45 patients had CA stroke, and 55 patients had lacunar and asymptomatic lesions. The majority of patients had AHA classification type IV-V and VI which presented as vulnerable plaques. Of 63 patients with mild to moderate stenosis ( $\leq 70 \%), 44(69.8 \%)$ had type IV-V
\end{abstract}

vulnerable plaques, which was significantly higher than those of patients with severe stenosis $(>70 \% ; p<0.001)$. In CA stroke, the number of patients with a thin or ruptured fibrous cap was twice that of those with a thick and intact fibrous cap. Conclusions: CEMRI may have important applications in clinical risk evaluations in CA atherosclerosis. Physicians ought to recognize that different types of stroke should be identified by brain MRI detection before invasive therapies.

Copyright $\odot 2009$ S. Karger AG, Basel

According to the reports of the WHO MONICA study, acute ischemic cerebrovascular disease of artherosclerosis remains the primary cause of morbidity and mortality in China [1]. Carotid atherosclerosis is closely correlated with subsequent stroke. About 23\% of ischemic stroke originates from carotid atherosclerosis and stenosis [2].

Nowadays, we generally recognize that predicting carotid artery (CA) vulnerable lesions is not possible by relying on stenosis alone. The low-grade disease of an atherosclerotic carotid can also result in cerebrovascular ischemic events [3]. We are becoming aware of the structure of the CA wall, including the composition, remodeling and inflammation of plaques. Rupture of the plaque

\section{KARGER}

Fax +4161306 1234 E-Mail karger@karger.ch www.karger.com
(C) 2009 S. Karger AG, Basel

$1015-9770 / 09 / 0274-0345 \$ 26.00 / 0$

Accessible online at:

www.karger.com/ced
Yongjun Wang, MD

Department of Neurology, Beijing Tiantan Hospital

Capital Medical University, No. 6 Tiantanxili

Chongwen District, Beijing 100050 (China)

Tel./Fax +86 106709 8350, E-Mail yongjunwang111@yahoo.com.cn 
surface and subsequent luminal thrombus formation are probably the most important mechanisms underlying acute ischemic stroke [4].

High-resolution contrast-enhanced MRI (CEMRI) is an optimal noninvasive imaging technique that allows the discrimination of large lipid cores with macrophage infiltration, thin and fragile fibrous caps, calcific nodules and adventitia in human atheromatous vulnerable plaques both in vivo and ex vivo [5]. This technique also characterizes intraplaque hemorrhage and acute thrombosis which fulfill the criteria for vulnerable plaque progression, stabilization and rupture [6].

Using a multiple CEMRI protocol, we matched the images of CA and the subsequent histological sections from specimens to verify the accuracy of MRI. Furthermore, we evaluated the relationship between carotid plaque characteristics and the different types of stroke in patients who had CA stenosis $\geq 50 \%$.

\section{Subjects and Methods}

\section{Study Population}

A single-center, prospective trial was conducted in the neurological department of Beijing Anzhen Hospital, Capital Medical University, from January 2007 to March 2008. We screened 102 hospitalized patients, 45 with acute ischemic stroke, including transient ischemic attacks (TIAs), who were subsequently proven to have $50-100 \%$ carotid stenosis by ultrasound, and 57 with lacunar and asymptomatic carotid stenosis, who sought medical treatment for their CA diseases. All patients underwent a detailed history assessment and a physical examination at baseline that included routine blood biochemistry tests, electrocardiography, chest radiography, ultrasound cardiography, transcranial Doppler sonography, carotid CEMRI, computed tomography, MRI and magnetic resonance angiography of the brain. Carotid and cerebral vascular digital subtraction angiography was performed once the patients' condition permitted the procedure.

We excluded patients who had (1) atrial fibrillation or a probable cardiac source of embolism, (2) Takayasu's arteritis, (3) intracranial artery stenosis proven by transcranial Doppler sonography and brain magnetic resonance angiography, and (4) any contraindication for MRI.

This study was approved by the Medical Ethical Committees of Beijing Tiantan Hospital, and written informed consent was obtained from each patient. The investigation conformed to the principles outlined in the Declaration of Helsinki.

All CA imaging and brain scans were reviewed collectively. Baseline brain MRI scans were evaluated to detect the types of brain infarction which corresponded to the territory of the index internal carotid artery (ICA).

The patients were divided into four categories according to the degree of stenosis measured by ultrasound: 50\%, 51-69\%, 70$89 \%$ and $90-100 \%$. In addition, the patients were assigned to 1 of 3 groups according to their results of brain MRI: (1) symptomatic group, lesion originated from the ICA, including watershed infarction (superficial-deep arterial and cortical border zone), mono- or multiembolic infarctions and TIA; (2) lacunar stroke, and (3) asymptomatic group.

TIA was defined as an onset focal neurological deficit lasting $<24 \mathrm{~h}$ with normal brain MRI. Lacunar strokes were defined by a combination of symptoms or signs and radiological criteria: presentation with deep white-matter lesions or basal-ganglion lesions $<15 \mathrm{~mm}$ in diameter [7]. An asymptomatic lesion was defined as producing no ipsilateral symptoms or signs caused by cerebral or retinal ischemia with normal brain MRI [8]

\section{MRI Protocol and Review}

All patients were imaged at the Beijing Anzhen Hospital in a 3.0T MRI Scanner (GE Signa, Excite, Waukesha, Wisc., USA), using an 8-channel phased-array CA coil. A standardized protocol was used to obtain 4 different contrast-weighted images - time of flight, $\mathrm{T}_{1}$-weighted, proton density-weighted and $\mathrm{T}_{2}$-weighted of the CAs centered at the common carotid bifurcation on the index side [9]. MRI parameters were: $\mathrm{T}_{1}$-weighted, double inversion recovery, black blood, 2-dimensional fast spin echo, repetition time/effective echo time $=800 / 11 \mathrm{~ms}$, echo train length $=12$; proton density-weighted and $\mathrm{T}_{2}$-weighted, fast-spin echo, cardiac gated, repetition time $=3$ or 4 cardiac R-R intervals, effective echo time $=15 \mathrm{~ms}$ for proton density-weighted and $65 \mathrm{~ms}$ for $\mathrm{T}_{2}$-weighted, echo train length $=12$, and 3-dimensional time of flight, repetition time/effective echo time $21 / 3.8 \mathrm{~ms}$, flip angle $15^{\circ}$. Images were obtained with a field of view of $14 \mathrm{~cm}$, matrix size of 256, slice thickness of $2 \mathrm{~mm}$.

Image quality was rated per artery for each contrast-weighted image on a 5 -point scale ( 1 = poor, 5 = excellent), and cases in which image quality was $\leq 2$ were excluded from the study. One experienced reviewer (W.Y.) who was blinded to clinical outcomes reviewed all magnetic resonance images for plaque features.

The tissue components including lipid-rich/necrotic core, calcification and hemorrhage were obtained by using a custom-designed Computer-Assisted System for Cardiovascular Disease Evaluation software [10]. The carotid bifurcation was used as a landmark for matching the 4 different contrast-weighted images at each slice location. The variety of tissue components was identified by using the criteria that had previously been proven in accordance with histology [11].

Fibrous cap status was categorized as either 'thick' versus 'thin or ruptured' based upon the previously published, histologically validated criteria [12]. The age of hemorrhage was classified as previously described [13].

\section{Histology Processing and Criteria}

Patients in the Department of Vascular Surgery underwent carotid endarterectomy (CEA) because of their atherosclerotic CA diseases. The excised intact specimens were fixed in $4 \%$ polyoxymethylene, decalcified and embedded in paraffin. Sections were cut (5 $\mu \mathrm{m}$ thick) in every $1.0 \mathrm{~mm}$ in the common CA and in every $0.5 \mathrm{~mm}$ in the bifurcation throughout the length of the specimen and stained (hematoxylin-eosin and Masson trichrome). Histological classification of specimens was made using the criteria of the modified American Heart Association (AHA) classification [14]. The slides were independently evaluated by a reviewer (X.F.H) who was unaware of the imaging results. 


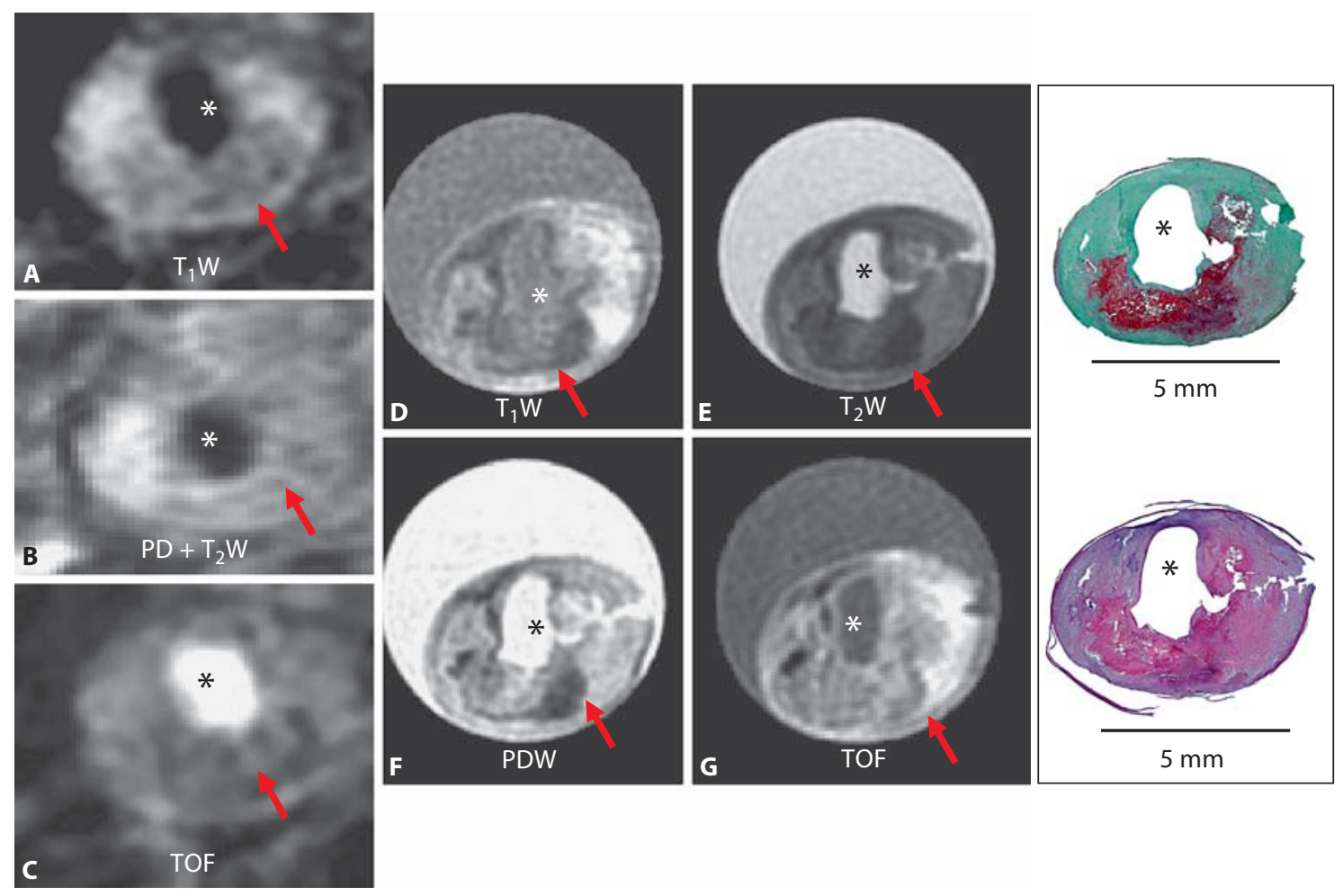

Fig. 1. Multicontrast weighted images $\left(\mathrm{T}_{1} \mathrm{~W}\right.$, TOF, $\mathrm{PDW}$ and $\left.\mathrm{T}_{2} \mathrm{~W}\right)$ of vulnerable plaque were obtained in vivo $(\mathbf{A}-\mathbf{C})$ and ex vivo (D-G). Asterisks indicate the lumen. Lumens show moderate eccentric stenoses. Tissue components of the wall: the thin fibrous cap above the large lipid core was enhanced; an old intraplaque hemorrhage adjacent to the lumen (arrows) produced hypointensity on MRI. Masson-trichrome- and hematoxylin-eosinstained matching histology sections showed good consistency of in vivo with ex vivo $M R I$. $\mathrm{T}_{1} \mathrm{~W}=\mathrm{T}_{1}$-weighted; $\mathrm{TOF}=$ time of flight; $\mathrm{PD}+\mathrm{T}_{2} \mathrm{~W}=$ proton density- and $\mathrm{T}_{2}$-weighted.

Criteria for defining vulnerable plaque was a thin or ruptured cap with a large lipid core, superficial platelet aggregation and lumen thrombosis, plaque surface ulceration and fissuration, intraplaque hemorrhage and a superficial calcified nodule [15].

\section{Statistical Analysis}

All data were expressed as means \pm SD. The Student unpaired $t$ test was used for comparison of enumeration data between 2 groups, and the Fisher exact probability test was used for comparison between categorical variables.

All calculations were made with SPSS 12.0 for Windows. Statistical significance was defined as a value of $\mathrm{p}<0.05$.

\section{Results}

\section{Carotid MRI and Specimen Validation Study}

The gross and histological morphology features came from 2 patients with CEA. The initial in vivo CEMRI and subsequent ex vivo imaging of specimens, including tissue components of lipid-rich/necrotic core, intraplaque hemorrhage and calcification, showed favorable consistency with histological findings. Histology and MRI matching images are shown in figures 1 and 2 .

\section{Patient Characteristics}

Of 102 consecutive patients (102 arteries) with atherosclerotic ICA disease, 100 patients (73 men and 27 women) met the criteria for an image quality rating of $\geq 3$. The mean age of the participants was $67.2 \pm 10.2$ years. The study demonstrated that 45 patients had CA stroke and 55 patients lacunar and asymptomatic infarction. Of 63 patients with stenosis $<70 \%$, 29 had stroke originating from the ICA. Of 37 with stenosis $\geq 70 \%, 16$ had ICA stroke. Patients with stenosis $<70 \%$ were more frequently seen with asymptomatic and lacunar stroke, but no significant differences between ICA and asymptomatic/lacunar stroke in mild and moderate-severe CA stenosis $(p=0.787)$ were found. The baseline clinical characteristics and baseline MRI findings are presented in table 1. 

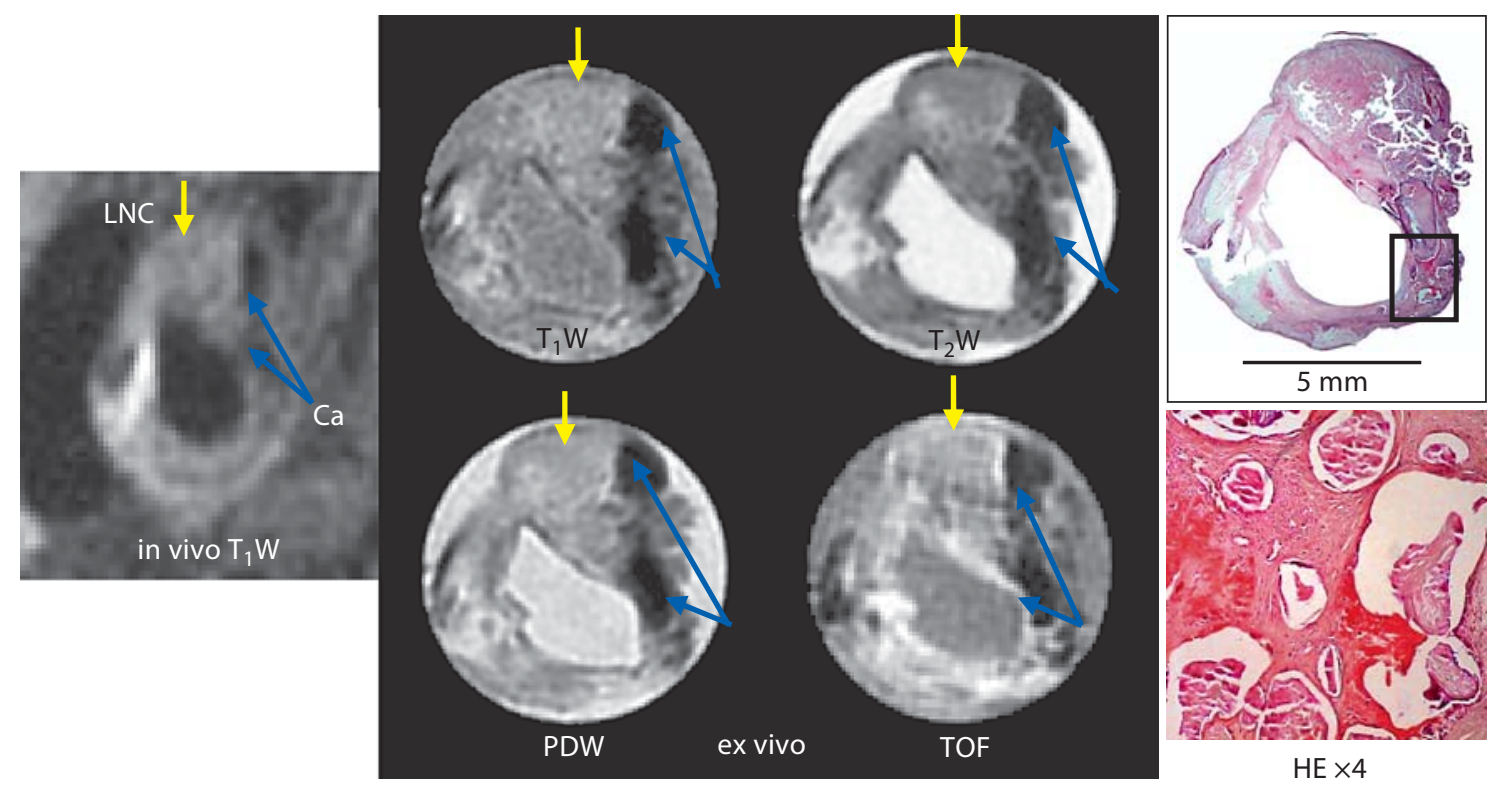

Fig. 2. CA bifurcation vulnerable plaque of in vivo and ex vivo samples as seen on MRI ( $T_{1} W$, TOF, PDW and $\mathrm{T}_{2} \mathrm{~W}$ ) along with the corresponding matched hematoxylin-eosin-stained (HE) histological cross-section. The external CA was seen just above and to the right of the ICA. The external CA was occluded with a large lipid necrotic core (LNC). Areas of dense calcification (Ca) appeared as a dark region from the 1- to 5-o'clock position on MRI and the histological cross-section of the ICA. The hematoxylin-eosin-stained matching histology section confirmed the presence of a superficial calcified nodule. $\mathrm{T}_{1} \mathrm{~W}=\mathrm{T}_{1}$-weighted; $\mathrm{TOF}=$ time of flight; $\mathrm{PDW}=$ proton density-weighted; $\mathrm{T}_{2} \mathrm{~W}=\mathrm{T}_{2}$-weighted.

Table 1. Baseline clinical characteristics ( $n=100$ patients)

\begin{tabular}{lccc}
\hline & $\begin{array}{l}\text { Watershed/ } \\
\text { embolism/TIAs } \\
(\mathrm{n}=45)\end{array}$ & $\begin{array}{l}\text { Asymptomatic/ } \\
\text { lacunar stroke } \\
(\mathrm{n}=55)\end{array}$ & $\begin{array}{l}\mathrm{p} \\
\text { value }\end{array}$ \\
\hline Age, year & $66.13 \pm 11.29$ & $68.00 \pm 9.30$ & 0.367 \\
Males & $33(73.3)$ & $40(72.7)$ & 1.000 \\
Smoking & $29(64.4)$ & $31(56.4)$ & 0.539 \\
Hypertension & $29(64.4)$ & $41(74.5)$ & 0.284 \\
Diabetes & $13(28.9)$ & $20(36.4)$ & 0.523 \\
Stenosis $<70 \%$ & $29(64.4)$ & $34(61.9)$ & 0.787 \\
Stenosis $\geq 70 \%$ & $16(35.6)$ & $21(38.2)$ & \\
TC, mmol/l & $4.63 \pm 0.98$ & $5.07 \pm 1.34$ & 0.068 \\
HDL-C, mmol/l & $1.20 \pm 1.03$ & $1.11 \pm 0.80$ & 0.253 \\
LDL-C, mmol/l & $3.13 \pm 0.94$ & $3.28 \pm 1.22$ & 0.253 \\
BG, mmol/l & $6.09 \pm 1.97$ & $6.89 \pm 3.51$ & 0.174 \\
hs-CRP, mg/l & $13.40 \pm 27.76$ & $4.82 \pm 8.36$ & 0.057 \\
\hline
\end{tabular}

$\mathrm{TC}=$ Total cholesterol; HDL-C = high-density lipoprotein cholesterol; LDL-C = low-density lipoprotein cholesterol; BG = blood glucose; hs-CRP = hypersensitivity C-reactive protein. Figures in parentheses indicate percentages.

\section{Vulnerable Plaques: MRI-Based Atherosclerotic Lesion Type Classification}

A total of 100 subjects were included by using the modified AHA classification: 4 cases of type III, which were equal to invulnerable plaques, which was defined as a diffuse intimal thickening or small eccentric plaque without calcification; 50 cases of type IV-V, which were similar to vulnerable plaques, which was described as plaque with a lipid or necrotic core surrounded by fibrous tissue with possible calcification; 43 cases of type VI represented complex vulnerable plaques with a possible surface defect, hemorrhage or thrombus; and 3 cases of type VII indicated calcified plaques. The majority of patients had AHA classification type IV-V and VI which presented as vulnerable plaques. There were no differences of plaque types among any category of patients.

\section{ICA Stenosis Degree and Types of Plaques}

Of 63 patients with mild to moderate stenosis $(\leq 70 \%)$, $44(69.8 \%)$ had type IV-V vulnerable plaques; this number was significantly higher than that of patients with severe stenosis $(>70 \%$; $<<0.001)$. Type VI was seen in most of the patients with severe stenosis. The relationship 
Fig. 3. Patient, male, 72 years. A Digital subtraction angiography (DSA) appearance of the left ICA with stenoses of $60 \%$. B Cervical B ultrasound showed 76\% left ICA stenoses with superficial ulceration. C Brain MRI: diffusion-weighted imaging indicated an infarction of the left corona radiata. D Contrast-enhanced magnetic resonance angiography (CEMRA) appearance of the left ICA with stenoses of $70 \%$. Plaque type with ruptured fibrous cap and intraplaque hemorrhage, delineation of ICA adventitia (green contour), lipid/necrotic core (yellow contour), intraplaque hemorrhage (pink contour) and lumen (red contour) shown in $\mathrm{T}_{1}$-weighted $\left(\mathrm{T}_{1} \mathrm{~W}\right)$, contrast-enhanced $\mathrm{T}_{1} \mathrm{~W}\left(\mathrm{CE} \mathrm{T}_{1} \mathrm{~W}\right)$, proton density-weighted (PDW) and $\mathrm{T}_{2}$-weighted $\left(\mathrm{T}_{2} \mathrm{~W}\right)$ images.

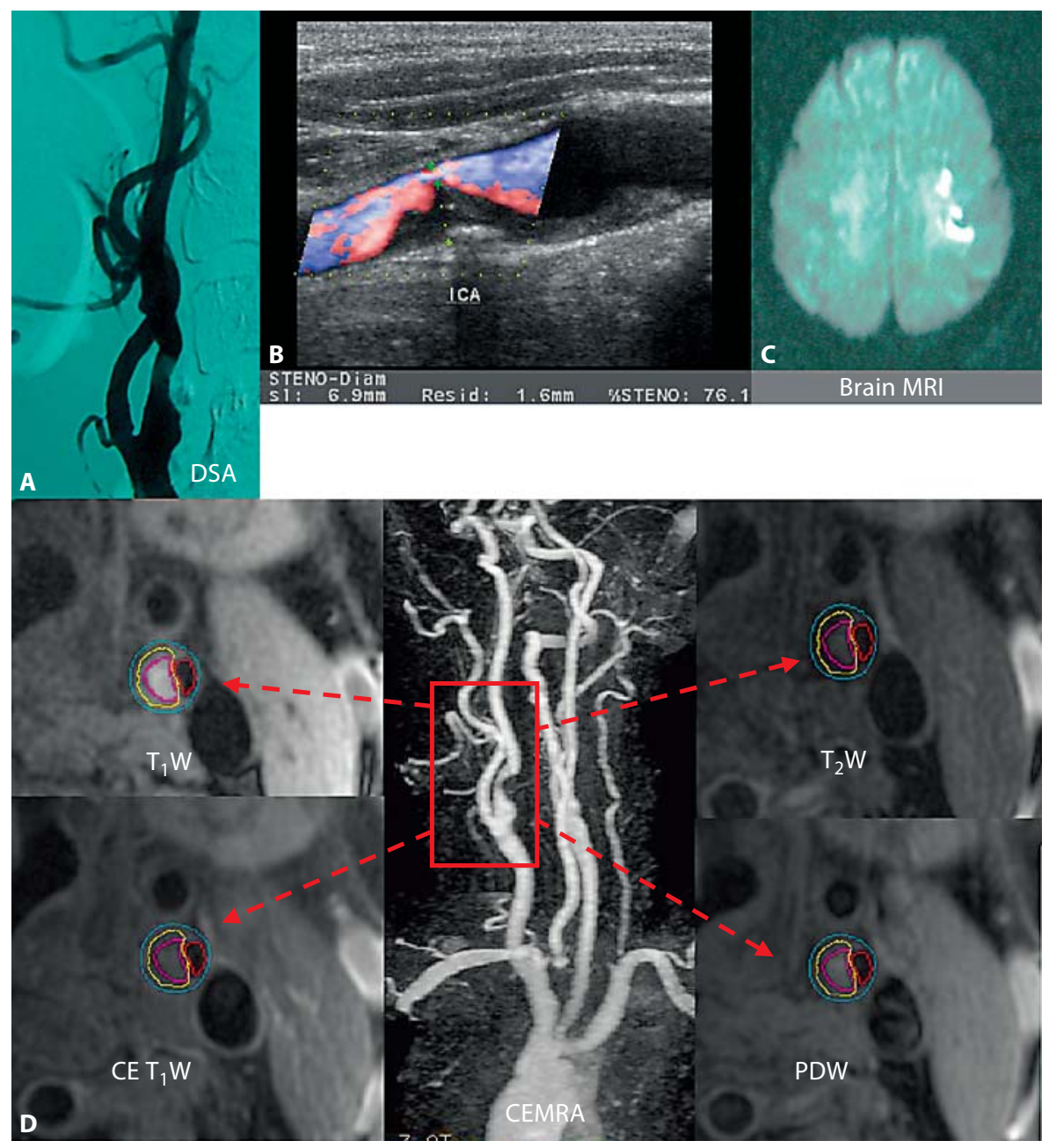

of different stenoses and types of plaques is shown in table 2 .

\section{Fibrous Cap Status and Intraplaque Hemorrhage}

Twenty-eight patients were observed to have a thick and intact (type I) superficial fibrous cap of the lumen. Thin or ruptured fibrous caps (type II) were observed in 72 subjects of whom 1 patient had luminal thrombosis. In CA stroke, the number of patients with thin or ruptured fibrous caps was twice that of those with thick and intact fibrous caps (30/15). Forty-six patients had intraplaque hemorrhage which was adjacent to the luminal surface. No differences of fibrous cap status and intraplaque hemorrhage were found in all groups. Figure 3 demonstrates serial imaging of one subcortical infarction patient with ICA vulnerable plaque.
Table 2. Relationship of different ICA stenoses and types of plaque

\begin{tabular}{llcccc}
\hline \multirow{2}{*}{ Stenosis } & \multicolumn{2}{l}{ Type } & & \\
\cline { 2 - 6 } & III & IV-V & VI & VII & total \\
\hline $50 \%$ & 3 & $27^{*}$ & 2 & 1 & 33 \\
$51-69 \%$ & & $17^{*}$ & 11 & 2 & 30 \\
$70-89 \%$ & 6 & 12 & 0 & 18 \\
$90-100 \%$ & 1 & 0 & 18 & 0 & 19 \\
\hline Total & 4 & 50 & 43 & 3 & 100 \\
\hline \multirow{2}{*}{$* \mathrm{p}<0.001}$. & & & & & \\
\hline
\end{tabular}




\section{Discussion}

Results of the North American Symptomatic Carotid Endarterectomy Trial indicate that the benefit of CEA in stroke patients with symptomatic stenosis is $>69 \%$ [16]. However, patients who have vulnerable lesions, such as those with low-grade stenosis, may also experience cerebrovascular events. Pathological studies suggest that the atherosclerotic plaque composition may represent an independent risk factor for ischemic stroke.

Rupture-prone lesions or vulnerable plaques consist of a large atheromatous core, which is separated from the lumen by a thin fibrous cap. Inflammatory cells such as macrophages and Tlymphocytes are located in the 'shoulder' region of the fibrous cap which lacks smooth muscle cells and collagen [17].

As the focus of CA studies has been shifted from stenosis to composition, imaging modalities that can distinguish plaque components are coming to the forefront. A series of experiments involving ex vivo imaging of endarterectomy specimens were carried out, and tissue components including lipid-rich necrotic core, hemorrhage and calcification were detected with a sensitivity and specificity ranging from 84 to $100 \%$. Translation of these findings to in vivo imaging showed that soft plaques (necrotic core or hemorrhage) could be identified with a sensitivity of $85 \%$ and a specificity of $92 \%$ [18].

Histological findings from this study further demonstrated that CEMRI permitted depiction of the vessel adventitial boundary and that it was also capable of identifying different components of vulnerable plaques. Carotid plaques excised from CEA patients presented the typical pathological characteristics of more advanced atherosclerotic vessel lesions. However, the amount of carotid plaque sections was limited, it was not enough to verify the accuracy of CEMRI. In a further study, we intend to find more CA specimens for MRI detection, and interrater and intrarater reliability assessments will be performed.

The vulnerable plaque plays an important role in critical events of stroke pathogenesis. Morphological data from autopsies as well as clinical MRI studies indicate the presence of surface irregularities, which has been proven highly associated with a recent history of TIA and stroke $[19,20]$.

After excluding patients with probable cardiac origin embolism and intracranial artery stenosis detected by ultrasound cardiography, transcranial Doppler sonography and brain magnetic resonance angiography, we subsequently enrolled the majority of patients $(63 \%)$ with mild to moderate stenosis $(<70 \%)$. The asymptomatic and lacunar stroke cases (34\%) were mostly seen in this group. Different categories of stroke, especially the topographic patterns of infarction associated with ICA stenosis, have been widely described in previous studies [21, 22]. Former studies indicated that in patients with symptomatic and asymptomatic stenosis, approximately 20 $40 \%$ of subsequent ipsilateral strokes were attributable to lacunes and cardioembolism separately [23]. Our study agreed with this conclusion. As the result, the decisions about whether to recommend CEA for such patients must be taken into account. Not all future strokes will originate from the stenosed ICA.

However, the degree of stenosis does not always accurately point out those patients who will eventually develop vulnerable lesions, because low-grade stenosis with vulnerable lesions may also result in cerebrovascular events [24]. In the present study, the association between baseline CA plaque features and stenosis showed that in moderate and severe ICA stenosis ( $\geq 70 \%)$, the number of patients with advanced plaques of AHA classification type VI was higher than that of patients with stenosis $<70 \%$, and plaque type IV-V was frequently seen in patients with stenosis $<70 \%$.

Virmani et al. [24] indicated that thin cap fibroatheroma, which was equal to AHA classification type IV-V, was a vulnerable plaque characterized by a necrotic core with an overlying thin fibrous cap $(<65 \mu \mathrm{m})$. The thin cap fibroatheroma usually occurred in lesions showing $<50 \%$ diameter stenosis; it had been postulated to be the precursor lesion of plaque ruptures and was more frequently observed in stroke patients [25]. The complicated type VI was a ruptured plaque with surface defects, hemorrhage and thrombosis. This kind of lesions in severe CA stenosis was subject to higher blood flow of the carotid bifurcation region. The high shear stress promoted the rupture of the thin fibrous cap. The high pressure in the lumen also induced rupture of the intraplaque vasa vasorum and intraplaque hemorrhage, and the atheromatous plaque might quickly develop into a fissure. Furthermore, rupture of the fibrous cap, with the resultant exposure of thrombogenic subendothelial plaque constituents, was believed to be the critical event that leads to thromboembolic complications [26].

CA plaque AHA classification types IV-V and VI, which were equal to vulnerable plaque, frequently occurred in all kinds of patients. No distinction was found in ICA and asymptomatic/ lacunar stroke. The focus of studies on symptomatic high-grade stenosis lesions and asymptomatic autopsy specimens without high-grade 
CA stenosis showed that high-grade carotid stenotic plaques were associated with a significantly higher incidence of complicated plaques compared with nonstenotic asymptomatic plaques. Nevertheless, complicated plaques were also present in moderate stenosis, and they did not distinguish between symptomatic endarterectomy and asymptomatic autopsy lesions [27]. Most of our patients had mild stenosis (63\%). There were no distinct morphological differences between asymptomatic and symptomatic carotid lesions.

We here investigated the superficial fibrous cap morphology of CA and its relationship with different kinds of stroke. In CA stroke, the number of patients with a thin or ruptured fibrous cap was twice that of patients with a thick and intact fibrous cap. It is well known that a thin or ruptured fibrous cap is an important morphological feature of the vulnerable atherosclerotic plaque. Patients with thin and ruptured fibrous caps were more likely to have a recent TIA or stroke [19].

In our study, thin and ruptured fibrous caps were also found in patients with asymptomatic and lacunar strokes. Morphological studies of coronary arteries suggested that plaque progression beyond $50 \%$ cross-sectional luminal narrowing occurred secondary to repeated ruptures, which might be clinically silent. The sites of healed plaque ruptures could be recognized by a necrotic core with a discontinuous fibrous cap [28].

As the causes and risk of stroke in patients with carotid vulnerable plaques are complicated, we must take into account that not all future strokes will originate from the vulnerable ICA. In our study, vulnerable plaques (type IV-V and VI) frequently occurred in all kinds of stroke patients; thin and ruptured fibrous caps were also found in patients with asymptomatic and lacunar lesions. This indicated that such patients might have arteriosclerosis of the intracranial circle of Willis and even arteriolosclerosis combined with CA disease. CA stroke that arises from a rupture or fissure (small rupture) of a vulnerable plaque may contribute to the natural history of plaque progression and ultimately luminal stenosis; the plaque tissue components became more complicated (type IV-V and VI) in which a lipid-rich necrotic core, hemorrhage and calcification could be detected. In general, a vulnerable atherosclerotic plaque of the stenotic $\mathrm{CA}$ is the underlying cause of the majority of ischemic strokes, and specific plaque characteristics have been associated with ischemic brain injury.

In conclusion, this prospective study indicates that high-resolution CEMRI may provide a useful tool for risk stratification and selection of candidates for invasive therapies. The alternative therapeutic agents which can initiate actual plaque regression have created a need to attempt to image the plaque itself, with the CA being an achievable target [29]. Atherosclerosis of the ICA is an important cause for stroke. Stroke patterns in ICA disease are complicated and include large-artery and lacunar stroke which is of small-artery origin. Physicians ought to recognize that the causes of stroke should be identified by brain MRI before invasive therapies.

\section{Acknowledgments}

We are grateful to Dr. Wei Wang and Dr. Xiaofen He, working in the Department of Pathology and Department of Neurology, for their kindly support to this research.

\section{References}

1 Thorvaldsen P, Kuulasmaa K, Rajakangas AM, et al: Stroke trends in the WHO MONICA project. Stroke 1997;28:500-506.

$\checkmark 2$ Hennerici M, Hulsbomer HB, Hefter H, et al: Natural history of asymptomatic extracranial arterial disease: results of a long-term prospective study. Brain 1987;110:777-791.

$\checkmark 3$ Wasserman BA, Wityk RJ, Trout HH 3rd, et al: Low-grade carotid stenosis: looking beyond the lumen with MRI. Stroke 2005;36: 2504-2513.

4 Carr S, Farb A, Pearce WH, et al: Atherosclerotic plaque rupture in symptomatic carotid artery stenosis. J Vasc Surg 1996;23:755765 .

Atherosclerotic Carotid Vulnerable

Plaque and Subsequent Stroke
5 Yuan C, Kerwin WS, Ferguson MS, et al: Contrast-enhanced high resolution MRI for atherosclerotic carotid artery tissue characterization. J Magn Reson Imaging 2002;15: 62-67.

6 Saam T, Hatsukami TS, Takaya N, et al: The vulnerable, or high-risk, atherosclerotic plaque: noninvasive MR imaging for characterization and assessment. Radiology 2007; 244:64-77.

7 Inzitari D, Lamassa M: Small-vessel disease with lacunes. Adv Neurol 2003;92:141-146. $\checkmark 8$ Inzitari D, Eliasziw M, Gates P, et al: The causes and risk of stroke in patients with asymptomatic internal-carotid-artery stenosis. North American Symptomatic Carotid Endarterectomy Trial Collaborators. N Engl J Med 2000;342:1693-1700.

$\checkmark 9$ Yuan C, Mitsumori LM, Beach KW, et al: Carotid atherosclerotic plaque: noninvasive MR characterization and identification of vulnerable lesions. Radiology 2001;221:285299.

10 Phan BA, Chu B, Kerwin WS, et al: Effect of contrast enhancement on the measurement of carotid arterial lumen and wall volume using MRI. J Magn Reson Imaging 2006;23: 481-485. 
11 Fayad ZA, Fuster V: Characterization of atherosclerotic plaques by magnetic resonance imaging. Ann NY Acad Sci 2000;902:173186.

-12 Hatsukami TS, Ross R, Polissar NL, et al: Visualization of fibrous cap thickness and rupture in human atherosclerotic carotid plaque in vivo with high-resolution magnetic resonance imaging. Circulation 2000;102:959964.

-13 Chu B, Kampschulte A, Ferguson MS, et al: Hemorrhage in the atherosclerotic carotid plaque: a high-resolution MRI study. Stroke 2004;35:1079-1084.

-14 Cai JM, Hatsukami TS, Ferguson MS, et al: Classification of human carotid atherosclerotic lesions with in vivo multicontrast magnetic resonance imaging. Circulation 2002; 106:1368-1373.

15 Naghavi M, Libby P, Falk E, et al: From vulnerable plaque to vulnerable patient: a call for new definitions and risk assessment strategies. Part I. Circulation 2003;108: 1664-1672.

16 North American Symptomatic Carotid Endarterectomy Trial Collaborators: Beneficial effect of carotid endarterectomy in symptomatic patients with high-grade carotid stenosis. N Engl J Med 1999;325:445-453.
17 Moreno PR, Falk E, Palacios IF, et al: Macrophage infiltration in acute coronary syndromes: implications for plaque rupture. Circulation 1994;90:775-778.

18 Yuan C, Kerwin WS, Yarnykh VL, et al: MRI of atherosclerosis in clinical trials. NMR Biomed 2006;19:636-654.

19 Virmani R, Burke AP, Kolodgie FD, et al: Vulnerable plaque: the pathology of unstable coronary lesions. J Interv Cardiol 2002;15 439-446.

20 Yuan C, Zhang SX, Polissar NL, et al: Identification of fibrous cap rupture with magnetic resonance imaging is highly associated with recent transient ischemic attack or stroke. Circulation 2002;105:181-185.

21 Tsiskaridze A, Devuyst G, de Freitas GR, et al: Stroke with internal carotid artery stenosis. Arch Neurol 2001;58:605-609.

22 Szabo K, Kern R, Gass A, et al: Acute stroke patterns in patients with internal carotid artery disease: a diffusion-weighted magnetic resonance imaging study. Stroke 2001;32: 1323-1329.
23 Inzitari D, Eliasziw M, Sharpe BL, et al: Risk factors and outcome of patients with carotid artery stenosis presenting with lacunar stroke. Neurology 2000;54:660-666.

24 Virmani R, Ladich ER, Burke AP, et al: Histopathology of carotid atherosclerotic disease. Neurosurgery 2006;59(suppl 3):S219227.

25 Redgrave JN, Lovett JK, Gallagher PJ, et al: Histological assessment of 526 symptomatic carotid plaques in relation to the nature and timing of ischemic symptoms: the Oxford Plaque Study. Circulation 2006;113:23202328.

26 Spagnoli LG, Mauriello A, Sangiorgi G, et al: Extracranial thrombotically active carotid plaque as a risk factor for ischemic stroke. JAMA 2004;292:1845-1852.

-27 Bassiouny HS, Davis H, Massawa N, et al: Critical carotid stenoses: morphologic and chemical similarity between symptomatic and asymptomatic plaques. J Vasc Surg 1989; 9:202-212.

$>28$ Burke AP, Kolodgie FD, Farb A, et al: Healed plaque ruptures and sudden coronary death: evidence that subclinical rupture has a role in plaque progression. Circulation 2001;103: 934-940

29 Gillard JH: Advances in atheroma imaging in the carotid. Cerebrovasc Dis 2007;24 (suppl 1):40-48. 